\title{
Spectral radius of weighted composition operators in $L^{p}$-spaces
}

\author{
by \\ KRZYSZTOF ZAJKOWski (Białystok)
}

\begin{abstract}
We prove that for the spectral radius of a weighted composition operator $a T_{\alpha}$, acting in the space $L^{p}(X, \mathcal{B}, \mu)$, the following variational principle holds:$$
\ln r\left(a T_{\alpha}\right)=\max _{\nu \in M_{\alpha, \mathrm{e}}^{1}} \int_{X} \ln |a| d \nu,
$$

where $X$ is a Hausdorff compact space, $\alpha: X \rightarrow X$ is a continuous mapping preserving a Borel measure $\mu$ with $\operatorname{supp} \mu=X, M_{\alpha, \mathrm{e}}^{1}$ is the set of all $\alpha$-invariant ergodic probability measures on $X$, and $a: X \rightarrow \mathbb{R}$ is a continuous and $\mathcal{B}_{\infty}$-measurable function, where $\mathcal{B}_{\infty}=\bigcap_{n=0}^{\infty} \alpha^{-n}(\mathcal{B})$. This considerably extends the range of validity of the above formula, which was previously known in the case when $\alpha$ is a homeomorphism.
\end{abstract}

1. Introduction. The spectral radius is an important characteristic of bounded operators. For many classes of operators it is an object of independent interest. It turns out that for weighted composition operators the logarithm of the spectral radius (the spectral exponent) convexly depends on the logarithms of their weights.

First we briefly recall some known results. Let $X$ be a Hausdorff compact space with Borel measure $\mu$ and $\alpha: X \rightarrow X$ be a continuous mapping preserving $\mu$ (i.e. $\mu \circ \alpha^{-1}=\mu$ ). The operator $T_{\alpha} f=f \circ \alpha$ is a linear isometry in $L^{p}(X, \mu), 1 \leq p \leq \infty$. Let $a$ be a continuous function on $X$. Under the assumptions that $\alpha$ is a homeomorphism and $\operatorname{supp} \mu=X$, for the spectral radius $r\left(a T_{\alpha}\right)$ of the weighted composition operator

$$
\left(a T_{\alpha}\right) u(x)=a(x) u(\alpha(x))
$$

the following formula holds:

$$
\ln r\left(a T_{\alpha}\right)=\max _{\nu \in M_{\alpha, \mathrm{e}}^{1}} \int_{X} \ln |a| d \nu
$$

2010 Mathematics Subject Classification: Primary 47B37; Secondary 47A10, 44A15. Key words and phrases: spectral radius, weighted composition operators, conditional expectation operators, Perron-Frobenius operators, Legendre-Fenchel transform. 
where $M_{\alpha, \mathrm{e}}^{1}$ is the set of all $\alpha$-invariant ergodic probability measures on $X$. The above formula was originally pointed out by Antonevich and proved in a number of concrete situations for example in [2, 3]. In the general form (for every homeomorphism $\alpha$ ) the formula was independently established by Kitover [10] and Lebedev [14].

If $\alpha$ is not invertible, then in $C(X)$ we have the same formula, while the case of $L^{p}(X, \mu)$ is more complicated. If $\alpha$ is a Markov shift then Stepin and Latushkin [12, 13] have obtained the formula

$$
\ln r\left(a T_{\alpha}\right)=\sup _{\nu \in M_{\alpha}^{1}}\left\{\int_{X} \ln |a| d \nu-\frac{1}{p}\left(\int_{X} \rho d \nu-h_{\alpha}(\mu)\right)\right\},
$$

where $h_{\alpha}$ is the metric entropy and $\rho$ is a certain function defined in terms of the map $\alpha$.

In the general case of an arbitrary continuous and noninvertible map $\alpha$ Antonevich, Bakhtin and Lebedev constructed a functional $\tau_{\alpha}$, called $T$ entropy, on the set $M_{\alpha}^{1}$ of $\alpha$-invariant probability measures such that

$$
\ln r\left(a T_{\alpha}\right)=\max _{\nu \in M_{\alpha}^{1}}\left\{\int_{X} \ln |a| d \nu-\frac{\tau_{\alpha}(\nu)}{p}\right\} .
$$

For an $\alpha$-invariant probability measure $\nu$ the T-entropy is defined as follows:

$$
\begin{gathered}
\tau_{\alpha}(\nu)=\inf _{n \in \mathbb{N}} \frac{\tau_{n}(\nu)}{n}, \quad \tau_{n}(\nu)=\inf _{D} \tau_{n}(\nu, D), \\
\tau_{n}(\nu, D)=\sup _{m \in M^{1}} \sum_{g \in D} \nu(g) \ln \frac{m\left(E_{\alpha^{n}} g\right)}{\nu(g)} .
\end{gathered}
$$

Here $D$ denotes a continuous partition of unity on $X$ and $E_{\alpha^{n}}$ is the conditional expectation operator with respect to the $\sigma$-algebra generated by $\alpha^{n}$. The above result was announced in [6, 5] and its proof can be found in [7]. Notice the functional $\tau_{\alpha}$ is nonnegative, convex and lower semicontinuous on $M_{\alpha}^{1}$.

For positive $a \in C(X)$ let $\varphi=\ln a$. Then the functional $\lambda(\varphi)=\ln r\left(e^{\varphi} T_{\alpha}\right)$ is continuous and convex on $C(X)$ (see [7]). The formula (1.2) means that $\lambda$ is the Legendre-Fenchel transform of the function $\tau_{\alpha} / p$, i.e. $\lambda^{*}=\tau_{\alpha} / p$ and its effective domain $D\left(\lambda^{*}\right)$ is contained in $M_{\alpha}^{1}$.

In this paper we prove that formula (1.1) holds in a more general setting, namely when the weight $a$ is continuous and $\mathcal{B}_{\infty}$-measurable, where $\mathcal{B}_{\infty}=\bigcap_{n=0}^{\infty} \alpha^{-n}(\mathcal{B})$ and $\mathcal{B}$ is the $\sigma$-field of Borel subsets of $X$. For any homeomorphism $\alpha$ the $\sigma$-fields $\mathcal{B}_{\infty}$ and $\mathcal{B}$ coincide and any continuous $a$ is also $\mathcal{B}_{\infty}$-measurable.

2. Legendre-Fenchel transform. The definition of the LegendreFenchel transform and its properties can be found for instance in [9]. Let 
$f$ be a functional on a real locally convex Hausdorff space $L$ with values in $\overline{\mathbb{R}}=[-\infty,+\infty]$. The set $\{\varphi \in L: f(\varphi)<+\infty\}$ is called the effective domain of the functional $f$. Let $L^{*}$ be the dual space to $L$. The functional $f^{*}: L^{*} \rightarrow \overline{\mathbb{R}}$ defined on the dual space by

$$
f^{*}(\mu)=\sup _{\varphi \in L}\{\langle\mu, \varphi\rangle-f(\varphi)\}=\sup _{\varphi \in D(f)}\{\langle\mu, \varphi\rangle-f(\varphi)\}, \quad \mu \in L^{*},
$$

is called the Legendre-Fenchel transform of $f$ (or the convex conjugate of $f$ ). The Legendre-Fenchel transform of $f^{*}$ is the functional on the initial space given by the similar formula:

$$
f^{* *}(\varphi)=\sup _{\mu \in L^{*}}\left\{\langle\mu, \varphi\rangle-f^{*}(\mu)\right\}, \quad \varphi \in L .
$$

Notice that $f^{*}$ is convex and lower semicontinuous with respect to the weak-* topology on the dual space. Moreover, if $f: L \rightarrow(-\infty,+\infty]$ is not identically equal to $+\infty$ and is convex and lower semicontinuous then $f^{* *}=f$, i.e. the Legendre-Fenchel transform is involutory.

It will be important for us to compare the Legendre-Fenchel transform on the initial space $L$ to itself defined on some subspace $W \subset L$. Elements of $W^{*}$ can be considered as equivalence classes of the following relation in $L^{*}$ :

$$
\mu \sim \nu \Leftrightarrow \forall_{\varphi \in W}\langle\mu, \varphi\rangle=\langle\nu, \varphi\rangle .
$$

For $[\mu] \in\left(L^{*} / \sim\right) \simeq W^{*}$ and $\varphi \in W$ the value of $[\mu]$ at $\varphi$ is given by

$$
\langle[\mu], \varphi\rangle=\langle\mu, \varphi\rangle \quad \text { for any } \mu \in[\mu] .
$$

Let $\widetilde{f}=\left.f\right|_{W}$. The Legendre-Fenchel transform of $\widetilde{f}$ is

$$
\tilde{f}^{*}([\mu])=\sup _{\varphi \in W}\{\langle[\mu], \varphi\rangle-\widetilde{f}(\varphi)\}=\sup _{\varphi \in W}\{\langle\mu, \varphi\rangle-f(\varphi)\} \quad \text { for any } \mu \in[\mu] .
$$

The connection between $f^{*}$ and $\widetilde{f}^{*}$ is given below.

Theorem 2.1. Let $L$ be a locally convex Hausdorff space and $f: L \rightarrow$ $(-\infty,+\infty]$ be a convex and lower semicontinuous functional not identically equal to $+\infty$. Let $W$ be a linear subspace of $L$ and set $\left.f\right|_{W}=\tilde{f}$. Suppose that the effective domain $D\left(f^{*}\right)$ is weak-* compact. Then

$$
\tilde{f}^{*}([\mu])=\min _{\mu \in[\mu]} f^{*}(\mu) \quad \text { for }[\mu] \in W^{*} .
$$

Proof. Because $L^{*}=\bigcup_{[\mu] \in W^{*}}[\mu]$ and $f=f^{* *}$, for $\varphi \in L$ we get

$$
f(\varphi)=\sup _{\mu \in L^{*}}\left\{\langle\mu, \varphi\rangle-f^{*}(\mu)\right\}=\sup _{[\mu] \in W^{*}} \sup _{\mu \in[\mu]}\left\{\langle\mu, \varphi\rangle-f^{*}(\mu)\right\} .
$$

If $\varphi \in W$ then $f(\varphi)=\widetilde{f}(\varphi)$ and $\langle\mu, \varphi\rangle=\langle[\mu], \varphi\rangle$, so the above equality can be rewritten as

$$
\widetilde{f}(\varphi)=\sup _{[\mu] \in W^{*}} \sup _{\mu \in[\mu]}\left\{\langle[\mu], \varphi\rangle-f^{*}(\mu)\right\}=\sup _{[\mu] \in W^{*}}\left\{\langle[\mu], \varphi\rangle-\inf _{\mu \in[\mu]} f^{*}(\mu)\right\} .
$$


To prove that $\tilde{f}^{*}([\mu])=\min _{\mu \in[\mu]} f^{*}(\mu)$ we must show that $\inf _{\mu \in[\mu]} f^{*}(\mu)$ is attained on $[\mu]$ and that this infimum is convex and lower semicontinuous on $W^{*}$.

Let $\mu, \nu \in L^{*}$. For any $\epsilon$ there exist $\mu^{\prime} \in[\mu]$ and $\nu^{\prime} \in[\nu]$ such that $f^{*}\left(\mu^{\prime}\right) \leq \inf _{\nu^{\prime} \in[\nu]} f^{*}(\nu)+\epsilon$ and $f^{*}\left(\nu^{\prime}\right) \leq \inf _{\mu^{\prime} \in[\mu]} f^{*}(\mu)+\epsilon$. By convexity of $f^{*}(\mu)$ one gets

$$
\begin{aligned}
t \inf _{\mu \in[\mu]} f^{*}(\mu)+(1-t) \inf _{\nu \in[\nu]} f^{*}(\nu)+\epsilon & \geq t f^{*}\left(\mu^{\prime}\right)+(1-t) f^{*}\left(\nu^{\prime}\right) \\
& \geq f^{*}\left(t \mu^{\prime}+(1-t) \nu^{\prime}\right) \\
& \geq \inf _{t \mu+(1-t) \mu \in[t \mu+(1-t) \nu]} f(t \mu+(1-t) \mu) .
\end{aligned}
$$

Now we prove that $\inf _{\mu \in[\mu]} f^{*}(\mu)$ is lower semicontinuous on $W^{*}$, that is, the set $\widetilde{D}_{c}=\left\{[\mu] \in W^{*}: \inf _{\mu \in[\mu]} f^{*}(\mu) \leq c\right\}$ is closed in the quotient topology for all $c \in \mathbb{R}$. Because $f^{*}$ is lower semicontinuous in the weak-* topology, for every $c \in \mathbb{R}$ the set $D_{c}=\left\{\mu \in L^{*}: f^{*}(\mu) \leq c\right\}$ is weak-* compact as a closed subset of the compact effective domain $D\left(f^{*}\right)$. Notice that if $\mu \in D_{c}$ then $[\mu] \in \widetilde{D}_{c}$. On the other hand if $[\mu] \in \widetilde{D}_{c}$ then

$$
\inf _{\mu \in[\mu]} f^{*}(\mu)=\min _{\mu \in[\mu] \cap D\left(f^{*}\right)} f^{*}(\mu)
$$

since $[\mu] \cap D\left(f^{*}\right)$ is weak-* compact and $f^{*}$ is lower semicontinuous. Hence there exists $\mu^{\prime} \in[\mu]$ such that $\min _{\mu \in[\mu]} f^{*}(\mu)=f^{*}\left(\mu^{\prime}\right) \leq c$, i.e. $\mu^{\prime} \in D_{c}$. In this way $\widetilde{D}_{c}$ is the image of the set $D_{c}$ under the canonical projection. By the definition of the quotient topology, $\widetilde{D}_{c}$ is compact and hence closed, so $\min _{\mu \in[\mu]} f^{*}(\mu)$ is lower semicontinuous on $W^{*}$. Since the Legendre-Fenchel transform is an involution, we obtain

$$
\inf _{\mu \in[\mu]} f^{*}(\mu)=\min _{\mu \in[\mu]} f^{*}(\mu)=\tilde{f}^{*}([\mu]) .
$$

3. Spectral radius of weighted composition operators. Calculating the spectral radius of operators we need to find the norm of their $n$th powers. For $f$ in $L^{p}(X, \mu)$ we have

$$
\left\|\left(a T_{\alpha}\right)^{n} f\right\|_{p}^{p}=\int_{X} \prod_{i=0}^{n-1}\left|a \circ \alpha^{i}\right|^{p}\left(T_{\alpha^{n}}|f|^{p}\right) d \mu=\int_{X} T_{\alpha^{n}}^{*}\left(\prod_{i=0}^{n-1}\left|a \circ \alpha^{i}\right|^{p}\right)|f|^{p} d \mu .
$$

Since $\operatorname{supp} \mu=X$ we have

$$
\left\|\left(a T_{\alpha}\right)^{n}\right\|=\left\|T_{\alpha^{n}}^{*}\left(\prod_{i=0}^{n-1}\left|a \circ \alpha^{i}\right|^{p}\right)\right\|_{L^{\infty}}^{1 / p} .
$$

This leads to formula 1.2 on the spectral radius of $a T_{\alpha}$ (see [4, 5]). 
It turns out that the conjugate to $T_{\alpha}$ is the Perron-Frobenius operator $P_{\alpha}$ (see [1]) defined as follows:

$$
\forall A \in \mathcal{B} \quad \int_{A} P_{\alpha} f d \mu=\int_{\alpha^{-1}(A)} f d \mu
$$

for $f$ in $L^{p}(X, \mu), 1 \leq p \leq \infty$ and $\mu \sigma$-finite. Let $\alpha^{-1}(\mathcal{B})$ denote the $\sigma$-algebra $\left\{\alpha^{-1}(A): A \in \mathcal{B}\right\}$. Notice that the $\alpha$-invariance of $\mu$ implies

$$
\int_{A} P_{\alpha} f d \mu=\int_{\alpha^{-1}(A)} T_{\alpha} P_{\alpha} f d \mu .
$$

Because $T_{\alpha} P_{\alpha} f=\left(P_{\alpha} f\right) \circ \alpha$ is an $\alpha^{-1}(\mathcal{B})$-measurable function and for every $B$ in $\alpha^{-1}(\mathcal{B})$,

$$
\int_{B} T_{\alpha} P_{\alpha} f d \mu=\int_{B} f d \mu
$$

the composition $T_{\alpha} P_{\alpha}$ is the conditional expectation operator $E(\cdot \mid \alpha)$ with respect to the $\sigma$-algebra $\alpha^{-1}(\mathcal{B})$ (see [8]). By properties of $E(\cdot \mid \alpha)$ and $\alpha$ invariance of $\mu$ we infer that for $f \in L^{p}(X, \mu)$ and $g \in L^{q}(X, \mu)(p, q$ are conjugate exponents),

$$
\begin{aligned}
\int_{X}\left(T_{\alpha} f\right) g d \mu & =\int_{X} E\left(\left(T_{\alpha} f\right) g \mid \alpha\right) d \mu=\int_{X}\left(T_{\alpha} f\right)\left(T_{\alpha} P_{\alpha} g\right) d \mu \\
& =\int_{X} f\left(P_{\alpha} g\right) d \mu .
\end{aligned}
$$

This proves that $P_{\alpha}$ coincides with $T_{\alpha}^{*}$. Moreover $P_{\alpha}$ is a left inverse to $T_{\alpha}$ since

$$
\forall_{A \in \mathcal{B}} \quad \int_{A} f d \mu=\int_{\alpha^{-1}(A)} T_{\alpha} f d \mu=\int_{A} P_{\alpha} T_{\alpha} f d \mu
$$

for all $f$ in $L^{p}(X, \mu)$.

ExAmple 3.1. Let $X=\mathbb{R} / \mathbb{Z} \simeq[0,1), \alpha(x)=n x(\bmod 1)$ and let $\mu$ be the Lebesgue measure on $X$. Obviously $\mu$ is $\alpha$-invariant. In this case the Perron-Frobenius operator has the form

$$
P_{\alpha}=\frac{1}{n} \sum_{i=0}^{n-1} T_{\beta_{i}}
$$

where $\beta_{i}(x)=(1 / n) x+i / n$. Notice that each $T_{\beta_{i}}$ is a left inverse to $T_{\alpha}$ but the left inverse which is conjugate to $T_{\alpha}$ has exactly the above form.

The proof of (1.1) for $\alpha$ a homeomorphism is based on the identity

$$
\left\|\left(a T_{\alpha}\right)^{n}\right\|=\left\|\prod_{i=0}^{n-1}\left(a \circ \alpha^{i}\right)\right\|_{\infty}=\max _{X} \prod_{i=0}^{n-1}\left|a\left(\alpha^{i}(x)\right)\right|
$$


(see [1, Th.5.1]). Now we will show that for $\alpha$ noninvertible, under some assumption on the weight $a$, the above formula still holds. In other words we exhibit a more general setting under which the spectral radius of the weighted composition operator is expressed by (1.1).

By the $\alpha$-invariance of $\mu$ and properties of $P_{\alpha}$ we can express (3.1) as

$$
\left\|\left(a T_{\alpha}\right)^{n}\right\|=\left\|T_{\alpha^{n}} P_{\alpha^{n}}\left(\prod_{i=0}^{n-1}\left|a \circ \alpha^{i}\right|^{p}\right)\right\|_{L^{\infty}}^{1 / p}=\left\|E\left(\prod_{i=0}^{n-1}\left|a \circ \alpha^{i}\right|^{p} \mid \alpha^{n}\right)\right\|_{L^{\infty}}^{1 / p} .
$$

To obtain 3.3 we should answer when $E\left(\prod_{i=0}^{n-1}\left|a \circ \alpha^{i}\right|^{p} \mid \alpha^{n}\right)=\prod_{i=0}^{n-1}\left|a \circ \alpha^{i}\right|^{p}$. By properties of the conditional expectation this holds when the function $\prod_{i=0}^{n-1}\left|a \circ \alpha^{i}\right|^{p}$ is $\alpha^{-n}(\mathcal{B})$-measurable.

Let $\mathcal{B}$ denote the Borel $\sigma$-algebra, $\mathcal{B}_{\infty}$ the $\sigma$-algebra $\bigcap_{n=1}^{\infty} \alpha^{-n}(\mathcal{B})$, and $C\left(X, \mathcal{B}_{\infty}\right)$ the space of all $\mathcal{B}_{\infty}$-measurable and continuous functions on $X$. If $a$ is $\mathcal{B}_{\infty}$-measurable then so is $a \circ \alpha^{i}$, and hence $\prod_{i=0}^{n-1}\left|a \circ \alpha^{i}\right|^{p}$ is $\alpha^{-n}(\mathcal{B})$ measurable for every $n$. In this way we obtain

TheOREM 3.2. Let $\alpha: X \rightarrow X$ be a continuous mapping preserving a measure $\mu$ on $X$ and $a \in C\left(X, \mathcal{B}_{\infty}\right)$. Then for the spectral radius of the weighted composition operator a $T_{\alpha}$ acting in $L^{p}(X, \mathcal{B}, \mu)$ the following variational principle holds:

$$
\ln r\left(a T_{\alpha}\right)=\max _{\nu \in M_{\alpha, \mathrm{e}}^{1}} \int_{X} \ln |a| d \nu,
$$

where $M_{\alpha, \mathrm{e}}^{1}$ is the set of all $\alpha$-invariant ergodic probability measures on $X$.

REMARK 3.3. Notice that if $\alpha$ is a homeomorphism of $X$ then $\mathcal{B}_{\infty}=\mathcal{B}$ and $C\left(X, \mathcal{B}_{\infty}\right)=C(X)$. In this case (1.1) holds for any continuous weight $a$. In this sense the above theorem is a generalization of the result obtained earlier by Antonevich, Kitover and Lebedev.

REMARK 3.4. For weights $a \in C\left(X, \mathcal{B}_{\infty}\right)$ formulae (1.1) and 1.2 are both valid, in the sense that the maximum in 1.2 is attained at $\nu$ for which $\tau_{\alpha}(\nu)=0$. In other words if $\tilde{\lambda}$ denotes the restriction of $\lambda$ to the subspace $C\left(X, \mathcal{B}_{\infty}\right)$ then $\tilde{\lambda}^{*}([\nu])=(1 / p) \min _{\nu \in[\nu]} \tau_{\alpha}(\nu)=0$ (see Th. 2.1p and this summand does not appear in 1.1.

Acknowledgements. I would like to thank Professor A. B. Antonevich for many valuable suggestions.

\section{References}

[1] A. B. Antonevich, Linear Functional Equations. Operator Approach, Minsk, Universitetskoe, 1988 (in Russian); English transl.: Birkhäuser, 1996. 
[2] A. B. Antonevich, A certain class of pseudodifferential equations with deviating argument on the torus, Diff. Uravneniya 11 (1975), 1550-1557 (in Russian); English transl.: Differential Equations 11 (1975).

[3] - Operators with a shift generated by the action of a compact Lie group, Sibirsk. Mat. Zh. 20 (1979), 467-478 (in Russian); English transl.: Siberian Math. J. 20 (1979), 329-337.

[4] A. B. Antonevich, V. I. Bakhtin and A. V. Lebedev, The variational principle for the spectral radius of weighted shift and weighted expectation operators, Dokl. Nats. Akad. Nauk Belarusi 2000, no. 6, 7-10 (in Russian).

[5] - , - - , Thermodynamics and spectral radius, Nonlinear Phenomena Complex Systems 4 (2001), 318-320.

[6] - , 一, - Spectra of operators associated with dynamical systems: from ergodicity to the duality principle, in: Twenty Years of Białowieża: a Mathematical Anthology, World Sci. Monogr. Ser. Math. 8, World Sci., Hackensack, NJ, 2005, 129-161.

[7] - - - - , T-entropy and variational principle for the spectral radius of transfer and weighted operators, arXiv:0809.3116v1 [math.DS], 18 Sep 2008.

[8] P. Billingsley, Probability and Measure, 3th ed., Wiley, New York, 1995.

[9] I. Ekeland and R. Temam, Convex Analysis and Variational Problems, NorthHolland, 1976.

[10] A. Kitover, The spectrum of automorphisms with weight, and the Kamowitz-Scheinberg theorem, Funktsional. Anal. i Prilozhen. 13 (1979), no. 1, 70-71 (in Russian); English transl.: Funct. Anal. Appl. 13 (1979), 57-58.

[11] A. Lasota and M. C. Mackey, Chaos, Fractals, and Noise. Stochastic Aspects of Dynamics, 2th ed., Appl. Math. Sci. 97, Springer, New York, 1994.

[12] Yu. D. Latushkin, On integro-functional operators with non-one-to-one shift, Izv. AN SSSR Ser. Mat. 45 (1981), 1241-1257 (in Russian); English transl.: Math. USSRIzv. (1981).

[13] Yu. D. Latushkin and A. M. Stepin, The weighted shift operators on a topological Markov chain, Funktsional. Anal. i Prilozhen. 22 (1988), no. 4, 86-87 (in Russian); English transl.: Funct. Anal. Appl. 22 (1988), 330-331.

[14] A. V. Lebedev, Invertibility of elements in $C^{*}$-algebras generated by dynamical systems, Uspekhi Mat. Nauk 34 (1979), no. 4, 199-200 (in Russian); English transl.: Russian Math. Surveys 34 (1979), no. 4, 174-175.

[15] A. M. Stepin, Homological equation in dynamical system theory, in: Studies in the Theory of Functions of Several Real Variables, Yaroslavl's State Univ., Yaroslavl, 1982, 106-117 (in Russian).

Krzysztof Zajkowski

Institute of Mathematics

University of Białystok

Akademicka 2

15-267 Białystok, Poland

E-mail: kryza@math.uwb.edu.pl

Received January 11, 2010

Revised version February 10, 2010 